\title{
The Lateral and Ventromedial Prefrontal Cortex Work as a Dynamic Integrated System: Evidence from fMRI Connectivity Analysis
}

\author{
Olivia Longe, Carl Senior, and Gina Rippon
}

\begin{abstract}
Recent functional magnetic resonance imaging (fMRI) investigations of the interaction between cognition and reward processing have found that the lateral prefrontal cortex (PFC) areas are preferentially activated to both increasing cognitive demand and reward level. Conversely, ventromedial PFC (VMPFC) areas show decreased activation to the same conditions, indicating a possible reciprocal relationship between cognitive and emotional processing regions. We report an fMRI study of a rewarded working memory task, in which we further explore how the relationship between reward and cognitive processing is mediated. We not only assess the integrity of reciprocal neural connections between the lateral PFC and VMPFC brain regions in different experimental contexts but also test whether additional cortical and subcortical regions influence this relationship. Psychophysiological interaction analyses were used as a measure of functional connectivity in
\end{abstract}

\section{INTRODUCTION}

Cognitive task performance in humans is often influenced by the motivational context in which it is performed. However, the precise manner in which motivational information influences higher-level cognitive processes and/or goal-directed behavior (Gilbert \& Fiez, 2004) at the neuronal level is uncertain. One emerging neuropsychological theory is that reward and cognitive systems must interface in order to achieve a goal (Gilbert \& Fiez, 2004; Taylor et al., 2004; Gray, Braver, \& Raichle, 2002; Pochon et al., 2002). Strong candidate regions for such an interface are the lateral prefrontal cortex (PFC), particularly the dorsolateral prefrontal cortex (DLPFC), and the ventromedial prefrontal cortex (VMPFC). DLPFC regions are thought to play a role in executive functions such as working memory and planning, whereas VMPFC regions are associated with reward sensitivity and motivation. Evidence for these regions providing a cognition/motivation interface has been suggested by nonhuman primate studies, which report that activity in DLPFC neurons correlates with motivational context (presence or absence of a reward) during

Aston University, Birmingham, UK order to characterize the influence of both cognitive and motivational variables on connectivity between the lateral PFC and the VMPFC. Psychophysiological interactions revealed negative functional connectivity between the lateral PFC and the VMPFC in the context of high memory load, and high memory load in tandem with a highly motivating context, but not in the context of reward alone. Physiophysiological interactions further indicated that the dorsal anterior cingulate and the caudate nucleus modulate this pathway. These findings provide evidence for a dynamic interplay between lateral PFC and VMPFC regions and are consistent with an emotional gating role for the VMPFC during cognitively demanding tasks. Our findings also support neuropsychological theories of mood disorders, which have long emphasized a dysfunctional relationship between emotion/motivational and cognitive processes in depression. working memory tasks (Watanabe, Hikosaka, Sakagami, \& Shirakawa, 2002). The impact of motivation on DLPFC activity may be driven by neurons in the VMPFC, especially as there is thought to be connectivity between these areas (Groenewegen \& Uylings, 2000; Schultz, Tremblay, \& Hollerman, 2000).

Recent functional magnetic resonance imaging (fMRI) studies have further sought to investigate the interaction between reward and cognitive performance by utilizing financially rewarded cognitive tasks. One consistent theme to emerge from these studies is that both increasing reward levels and cognitive task demands appear to correlate with an increased blood oxygenation level-dependent (BOLD) response in the DLPFC (BA 9/ 46) (Gilbert \& Fiez, 2004; Pochon et al., 2002) and lateral fronto-polar (BA 10) regions (Pochon et al., 2002). Conversely, increasing reward levels and cognitive task demands are reported to correlate with decreased activation in the anterior medial PFC (BA 10/11), subgenual cingulate cortices (BA 25) (Pochon et al., 2002), and ventral frontal areas (BA 11/47) (Gilbert \& Fiez, 2004; Pochon et al., 2002), suggesting a possible reciprocal relationship between lateral and medial PFC regions. An additional fMRI study employing an object-working memory task 
also reported an interaction between financial incentives (both rewards and punishments) and memory performance in the right DLPFC (Taylor et al., 2004), but did not report reward- or cognition-related decreases in medial prefrontal regions.

Some studies claim that task-induced decreases in medial PFC activity are the result of a baseline default mode of brain function, which becomes suspended during specific goal-directed behaviors (Raichle et al., 2001). Such baseline activity, according to Raichle et al. (2001), is usually involved in the emotional evaluation of information, but becomes attenuated when focused attention is required, in order to devote more resources to the task at hand. The same group proposes that changes in activation in the medial PFC during cognitive tasks may also be related to anxiety caused by the task (Simpson, Drevets, Snyder, Gusnard, \& Raichle, 2001; Simpson, Snyder, Gusnard, \& Raichle, 2001). An alternate viewpoint is that deactivations in this area serve a more general role in inhibiting emotional signals that interfere negatively with task performance, whether the emotion is anxiety, fear of failure, or even excitement (Pochon et al., 2002). This is supported by fMRI studies of reward and cognition interactions (Gilbert \& Fiez, 2004; Pochon et al., 2002), which report that increased task demands require the suppression of activity in medial PFC areas in order to reduce competing emotional responses to rewards that may interfere with task performance. In consequence, these studies suggest a form of "dynamic interplay" between regions responsible for cognitive function and regions responsible for affective processing. Such findings are additionally supported by neuropsychological theories of mood disorders, which have repeatedly underlined links between emotion and cognition. Most notably, Mayberg et al. (1999) suggested that a reciprocal relationship between the DLPFC and the ventral anterior cingulate (vACC) mediates the relationship between emotion and attention, and that this relationship is altered in depression (a disorder marked by reduced motivation, in addition to impaired memory and attention processing; for reviews see Phillips, Drevets, Rauch, \& Lane, 2003; Austin, Mitchell, \& Goodwin, 2001).

In the current study, we aim to further explore how the relationship between reward and cognitive processing is facilitated. As discussed above, the relationship may be facilitated through reciprocal neural connections between lateral PFC and VMPFC brain regions. However, the relationship between reward and cognitive processing could also be mediated by additional cortical and/or subcortical regions influencing the lateral PFC-VMPFC relationship, as both regions have strong anatomical connections with other cortical and subcortical sites known to have a role in reward processing and cognition. We therefore describe an fMRI study of a rewarded working memory task, in which we seek to understand the behavior of PFC regions highlighted, both in terms of levels of activity in isolated areas and interregional covariances, which are taken as an index of functional connectivity (White \& Alkire, 2003). We evaluate the impact of memory load in an $n$-back task, in addition to different reward manipulations in terms of levels of activity in lateral PFC areas [including the DLPFC (BA 9, 46) and the lateral fronto-polar cortex (BA 10/46; LatFPC)] and VMPFC areas [encompassing the vACC and the subgenual cingulate (BA 11/32/25)]. We also evaluate changes in integration between lateral and VMPFC areas, in addition to other regions of interest (which we expand on below), using psychophysiological interaction (PPI) analysis.

A PPI shows the change in the relationship of one brain region to another, in relation to an experimental manipulation of interest (Friston et al., 1997). The principle approach is an analysis that is a simplified form of a model-based connectivity analysis such as performed in structural equation modeling or dynamic causal modeling (e.g., Williams et al., 2006; Das et al., 2005; Egner \& Hirsch, 2005; Fletcher, McKenna, Friston, \& Dolan, 1999; McIntosh, Rajah, \& Lobaugh, 1999; Friston et al., 1997). This approach has an advantage over traditional fMRI analysis in providing information about the integration of physiological and experimental influences on brain activity (Das et al., 2005). As we anticipate reciprocal neural connections between lateral PFC and VMPFC regions, we expect that activity in the lateral PFC will show negative functional connectivity with the VMPFC in the context of an increased memory load manipulation and in the context of an increased reward manipulation.

Other cortical regions, particularly those associated with cognitive control, such as the dorsal anterior cingulate gyrus (dACC; Bush, Luu, \& Posner, 2000), may impact on reward/cognition interactions mediated by lateral PFC-VMPFC connections. Activation of the dACC is thought to help guide behavior by integrating information on motivation, target detection, reward encoding, and error signals in order to influence the allocation of attention and motor responses (Bush et al., 2000). The dACC is closely connected with the lateral PFC (BA 46/9), and when conflict is detected in a task, the dACC is thought to send a signal for controlled processing to the lateral PFC, which then leads individuals to engage in appropriate goal-directed behavior (Davidson, Pizzagalli, Nitschke, \& Putnam, 2002; Posner \& Raichle, 1994). In the current study, a finding of functional connectivity between the lateral PFC and the dACC in the context of increased reward level would suggest the dACC has a direct top-down influence on the lateral PFC. Such a direct effect may be the crucial signal that enables participants to suppress emotional signals in order to perform a working memory task. As a result, we would expect to find functional connectivity between the lateral PFC and the dACC in the context of reward but not specifically for memory load. In addition to psychophysiological analysis, we also carry out physiophysiological interaction analysis, 
which focuses on the physiological interaction between two brain regions and allows us to investigate whether activity between the lateral PFC and VMPFC regions is modulated by activity in other reward and cognitive processing regions. We expect a relationship between the dACC and the lateral PFC to be further evidenced in a physiophysiological interaction analysis, where we expect to find that the dACC modulates the lateral PFC-VMPFC pathway.

Finally, the relationship between affective and cognitive processing may additionally be mediated by corticalsubcortical connections. One likely candidate region is the dorsal striatum (i.e., the caudate), which has connections to the ventrolateral and dorsolateral PFC (Leh, Ptito, Chakravarty, \& Strafella, 2007; Yeterian \& Pandya, 1991) and may enable communication between different prefrontal regions. Neurons of the caudate are reported to encode visual cues that specifically predict reward, indicating this area in the processing of salient stimuli (Lauwereyns et al., 2002). This is confirmed by neuroimaging studies which have frequently implicated the caudate in processing a variety of rewards (Delgado, Locke, Stenger, \& Fiez, 2003; Elliott, Newman, Longe, \& Deakin, 2003; Berns, McClure, Pagnoni, \& Montague, 2001; Knutson, Adams, Fong, \& Hommer, 2001; Delgado, Nystrom, Fissell, Noll, \& Fiez, 2000; Elliott, Friston, \& Dolan, 2000). Based on the caudate's role in reward processing and connections with frontal association areas (Alexander, Crutcher, \& DeLong, 1990), variations in connectivity between this region and the lateral $\mathrm{PFC}$, in the context of variations in reward, may be observed. Additionally, if the caudate nucleus impacts on the relationship between the lateral PFC and the VMPFC, this will be further demonstrated in a physiophysiological interaction analysis where an interaction between the lateral PFC and the caudate nucleus would predict activity in the VMPFC.

\section{METHODS}

\section{Participants}

Ten healthy right-handed volunteers were recruited from the Aston University student population ( 2 men, 8 women; mean age $\pm S D=21.67 \pm 4.89$ years). Volunteers were screened for a history of neurological or psychiatric disease and those scoring over 10 on the Beck Depression Inventory (Beck et al., 1961) were also excluded. The purpose and risks of the study were explained to all volunteers, who gave written informed consent to participate, as approved by Aston University Ethics Committee.

\section{Experimental Design}

Participants performed a spatial $n$-back working memory task. Stimuli were presented in blocks of 12 trials. Each block had a duration of $42.5 \mathrm{sec}$ and began with the presentation of an instruction screen ( $4 \mathrm{sec}$ ) and ended with performance feedback (2 sec). Within a block, each trial started with a fixation spot $(500 \mathrm{msec})$, displayed in the center of a background configuration of randomly arrayed letters. This was followed by the presentation of a square box with several instances of the same letter inside, in uppercase or lowercase font (250 msec) (Figure 1). The box with letters was superimposed on the background of randomly arrayed letters. The box contained one of six possible letters and appeared in one of six possible locations. During the 2300-msec intertrial interval, the background of randomly arrayed letters was displayed alone. Participants had to indicate whether the spatial location of the box was the same ("target") or different ("nontarget") from that displayed either one or three trials ago (1-back and 3-back) by pressing a button on a response box. In addition, participants performed a control task, a 0-back task, which required participants to simply match the spatial location of each box displayed with the box that had been presented in the very first trial of the block.

To examine the relative effects of reward and working memory on the PFC network, two orthogonal factors were manipulated in the $n$-back task: (1) memory load and (2) reinforcement. Memory load was manipulated by using two different levels of the $n$-back task with 1-back placing a low load and 3-back a high load. The $n$-back task was performed in association with two different values of financial reward: $10 \mathrm{p}$ and £1. The task was also performed with no reinforcement present. The instruction screen prior to the start of each block displayed information regarding the type of task (0-back, 1-back or 3-back) and the rewarding value of the task (no money, $10 \mathrm{p}$, or £1). The experiment was divided into five runs of eight blocks (one each of the six experimental conditions and two control blocks). Run order was counterbalanced among participants.

Participants were informed that during rewarded blocks each correct response to a target would result in winning the amount at stake; however, each incorrect response to a target would result in losing that amount. Participants were also notified that they would receive the total amount won during the task at the end of the experiment. Average winnings were $\$ 13.50$ $(S D= \pm 5.37)$.

\section{Image Acquisition and Analysis}

fMRI was carried out on a 3-T Siemens Trio system at Aston University, using a T2*-weighted gradient-echo, echo-planar imaging sequence $(\mathrm{TR}=3 \mathrm{sec}, \mathrm{TE}=30 \mathrm{msec}$, flip angle $=90^{\circ}, \mathrm{FOV}=25 \times 25 \mathrm{~cm}$, matrix size $\left.=64 \times 64\right)$.

The images consisted of 40 axial slices (angled at $\sim 20^{\circ}$ away from the eyes, which allowed the slices to be angled away from the predominant direction of the intrinsic susceptibility-induced field gradients which can result in susceptibility and/or distortion artifacts in the ventral 


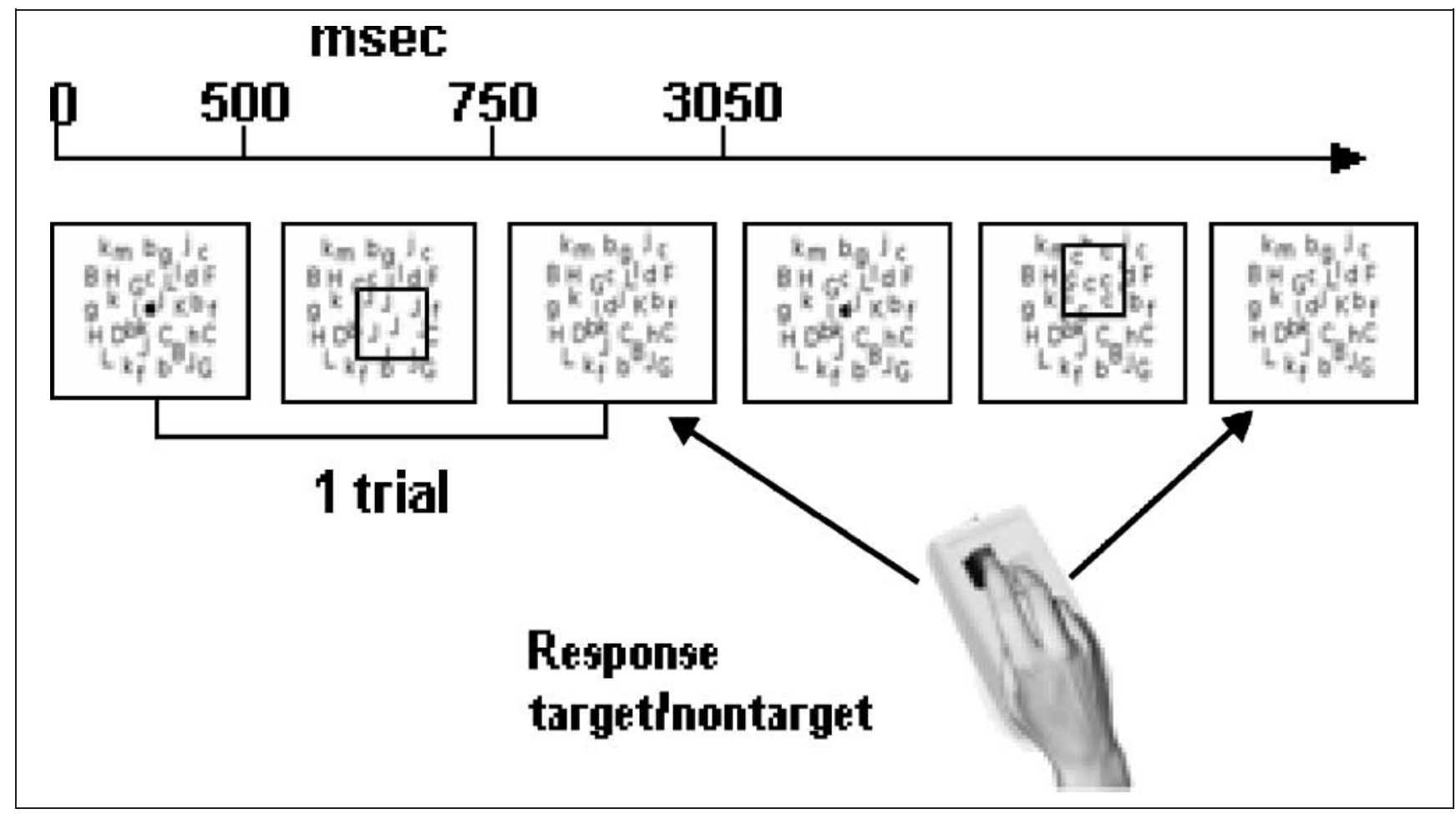

Figure 1. Schematic figure of the different events associated within a trial in the spatial $n$-back task. Each trial consisted of a fixation spot (500 msec) presented in the middle of randomly arrayed letters, followed by the presentation of a square box ( $250 \mathrm{msec}$ ), which also had randomly arrayed letters inside. The spatial location of the square box varied from trial to trial, and appeared in one of six locations. During the intertrial interval (2300 $\mathrm{msec})$, the background of randomly arrayed letters was displayed alone. In this period, participants indicated whether the box location was the same ("target") or different ("nontarget") from that displayed either one or three trials ago (1-back or 3-back).

PFC). Similarly, the relatively low TE value (above) was chosen to decrease potential signal dropout, as this results in less phase dispersion across voxels at high field strengths. Slices were $3 \mathrm{~mm}$ thick $(128 \times 128$ in-plane resolution). To allow equilibrium to reach steady state, two dummy volumes were collected before the start of each run (117 volumes) and discarded before analysis. T1weighted scans were acquired for anatomical localization.

Analysis was performed using SPM2 (Wellcome Institute of Neurology, implemented in Matlab; Mathworks, MA). Prior to model application, brain volumes from each participant were realigned to the first volume to correct for head motion. Functional images were then spatially normalized into a standard Montreal Neurological Institute (MNI) echo-planar imaging template. Following this, we applied spatial smoothing with an isotropic Gaussian kernel filter of $10-\mathrm{mm}$ full width at half maximum to facilitate intersubject averaging. Data were analyzed with a random effects model. For every participant, a single mean contrast image was produced for each comparison. The set of voxel values for each contrast constituted a statistical parametric map of the $t$ statistic $[\operatorname{SPM}(t)]$, which was then transformed to the unit normal distribution, $\operatorname{SPM}(Z)$. We thresholded the activations at a voxel threshold of $p<.001$, uncorrected, and accepted as significant those clusters that survived at $p<.05$, corrected for multiple comparisons for the entire brain. Because our main focus is on a prespecified network of regions outlined in the Introduction-the lateral PFC, the VMPFC, the fronto-polar cortex, the dACC, and the dorsal striatum-for these regions we report activations that survive an uncorrected threshold of $p<.001$, but are significant at $p<.05$ when a small volume correction (SVC) (based on a sphere of diameter $10 \mathrm{~mm}$, centered on the peak coordinates; Worsley et al., 1996) is applied. As SPM coordinates are given in MNI space, regions were identified by converting the coordinates to Talairach space with a nonlinear transform (Brett, Christoff, Cusack, \& Lancaster, 2001).

\section{fMRI Connectivity Analysis}

PPI analysis allows the detection of interactions between brain regions in relation to a study paradigm, that is, the way in which activity in one brain region modulates activity in another region specifically in response to a cognitive/sensory process (in this case, for high memory load relative to low and for high rewards relative to low or no reward) (Friston et al., 1997).

Using SPM2, we extracted for each individual the deconvolved time course of activity from a sphere of $6 \mathrm{~mm}$ radius centered on the most significant voxels from the group analyses identified as reflecting changes in memory load and changes in motivation in the lateral 
PFC (BA 10, 46 and BA 44) and the subgenual cingulate (BA 25). An SPM was then computed for each individual that revealed areas where activation is predicted by the PPI term. The individual contrast images were then taken to the second level to perform a random effect analysis (using a one-sample $t$ test). A stringent random effects model with a priori defined regions and a statistical threshold of $p<.005$ (uncorrected) was used, with an extent threshold of 7 voxels per cluster.

We also used a physiophysiological interaction analysis to examine how the interaction between lateral PFC and VMPFC regions varies with activation in the dACC and the caudate nucleus. This analysis focuses on the physiological interaction between two brain regions rather than the interaction between one brain region and the psychological impact of a task condition. We examined the effect of the interaction between the right lateral PFC and the right AACC and also between the right lateral PFC and the right caudate nucleus. Subjectspecific time series were extracted for each of these three regions, centered on the most significantly active voxel from the group analysis.

\section{RESULTS \\ Behavioral Results}

Reaction times were collected for each condition and the means were calculated. The data were analyzed in an analysis of variance (ANOVA) with two repeated measures (task type: 3-back or 1-back, and reward level). There was a significant difference between the three reward levels $[F(2,9)=13.57, p<.05]$. Post hoc analysis showed that this was due to faster reaction times in the low-reward 1-back task than in the no-reward 1-back task $[t(2,9)=3.541, p<.05]$ and also faster reaction times in the low-reward 1-back task compared to the high-reward 1-back task $[t(2,9)=3.457, p<.05]$. Also, as you would expect, there was a difference in reaction times between the task versions 1-back and 3-back (1-back responses faster than 3-back) $[F(2,9)=5.59$, $p<.05]$, but there was no interaction between task type and reward level $(F<1)$.

Mean percentage correct scores for each condition were also calculated. An ANOVA with two repeated measures (task type: 3-back or 1-back and reward level) showed that there was a significant difference in the percentage correct scores over the three reward levels $[F(2,9)=13.603, p<.05]$. Post hoc analysis showed that this was due to higher percentage correct scores in the high-reward than in the no-reward 1-back task $[t(2$, $9)=3.44, p<.05]$ and in the high-reward than in the low-reward 1-back task $[t(2,9)=5.81, p<.05]$. In addition, post hoc analysis showed that participants performed with more accuracy for high-reward compared to low-reward 3-back task $[t(2,9)=3.42, p<.05]$ and had greater accuracy for the high-reward compared to the no-reward 3-back task $[t(2,9)=2.21, p=.054]$. There was no interaction between task type and reward level.

\section{Imaging Results}

To aid in the interpretation of the principal analyses of interest, which are the psychophysiological and physiophysiological interactions, the results of the subtractive analysis are outlined first. For clarity, we only report activations within the prespecified network of regions we are testing with the PPI and physiophysiological analysis - the VMPFC (including the vACC and the subgenual cingulate), lateral $\mathrm{PFC}$ regions (including the DLPFC and the fronto-polar cortex), the dACC, and the caudate.

\section{Subtractive Analysis}

BOLD response to memory load. Similar to past studies assessing working memory, the contrast of 3-back compared to the control task (0-back; not reported in the tables) revealed significant activation in frontal areas commonly activated as part of a working memory network, including the right lateral fronto-polar cortex (LatFPC; BA 10), the left DLPFC (9/46; significant with SVC), and the superior frontal gyrus (BA 6, 8). The lower memory load of 1-back contrasted with control also revealed significant activation (with SVC) in the left LatFPC (BA 10) and the right LatFPC (BA 10 extending to 9; contrast not reported in the tables).

The main contrast of interest for assessing working memory load was the contrast of high-memory load (3-back) versus low memory load (1-back; Table 1). Areas displaying greater activation to high memory load included the right LatFPC (BA 10/46) and a cluster in the left LatFPC (BA 10), which extended ventrally to the orbitofrontal cortex (BA 11).

The reverse contrast of 1-back compared to 3-back assessed areas of reduced activation to memory load (Table 1; Figure 2). As hypothesized, we found that the vACC (BA 32) showed reduced activation to task difficulty. In addition, we observed reduced activation in the right medial PFC (BA 10), spreading to dorsomedial frontal regions (BA 8).

$B O L D$ response to reward. When we contrasted both high reward conditions compared to low rewards, we observed significant activation in the right dorsal ACC (BA 32/24), spreading laterally to the lateral PFC (BA 44), the right LatFPC (BA 10), which extended to the DLPFC (BA 46) (Table 2), and the left DLPFC (BA 46).

For the contrast of high rewards compared to norewards, we observed increased right caudate activation (Table 2). There was no significant PFC activation, although at a reduced threshold of $p<.01$, uncorrected, 
Table 1. Brain Areas Activated in the Contrast of: (A) All 3-back minus All 1-back; (B) All 1-back minus All 3-back

\begin{tabular}{|c|c|c|c|c|c|}
\hline Region & Cluster Size & p-Corrected & Brodmann's Area & Z Score & $x, y, z$ \\
\hline \multicolumn{6}{|l|}{ (A) All 3-back vs. All 1-back } \\
\hline \multirow[t]{2}{*}{ L LatFPC } & \multirow[t]{2}{*}{192} & \multirow[t]{2}{*}{$<.001$} & BA 10 & 4.18 & $-32,54,1$ \\
\hline & & & BA 10 & 3.44 & $-36,52,21$ \\
\hline L OFC & \multirow{3}{*}{267} & \multirow{3}{*}{.054} & BA 11 & 3.26 & $-30,52,-11$ \\
\hline \multirow[t]{2}{*}{ R LatFPC } & & & BA 10/46 & 4.15 & $30,57,18$ \\
\hline & & & BA 10 & 4.13 & $26,53,5$ \\
\hline \multicolumn{6}{|l|}{ (B) All 1-back vs. All 3-back } \\
\hline Midline vACC & 175 & $.015 *$ & BA 32 & 4.09 & $\mathbf{0}, 31,-8$ \\
\hline R Medial PFC & 190 & $.015 *$ & BA 10 & 3.51 & $10,53,10$ \\
\hline \multirow[t]{2}{*}{ R Dorsomedial frontal cortex } & & & BA $8 / 9$ & 3.50 & $12,50,38$ \\
\hline & & & BA $9 / 10$ & 3.38 & $4,54,21$ \\
\hline
\end{tabular}

Clusters significant at $p<.05$ after statistical correction are reported. Multiple peaks within an extent are shown on subsequent lines.

Coordinates are presented in Talairach space. $\mathrm{L}=$ left; $\mathrm{R}=$ right.

*Significantly corrected $p$ values shown after SVC.

there was right LatFPC (BA 10) activation. Additionally, examining this contrast at the simple effects level (not reported in the tables) for both the 3-back and 1-back task, we observed significant activation for high reward versus no-reward in the left lateral PFC (BA 44; -40, 5, 31), the right lateral PFC (BA 44; 40, 4, 33), the left
dACC (BA 24; - 16, 26, 22), and at a reduced cluster size (3 voxels) in the right DLPFC (BA 46; 44, 23, 25) to the 3-back task, but there was no significant PFC activation for the 1-back task.

Areas deactivated to rewards were assessed by comparing the main effects of unrewarded blocks to both low

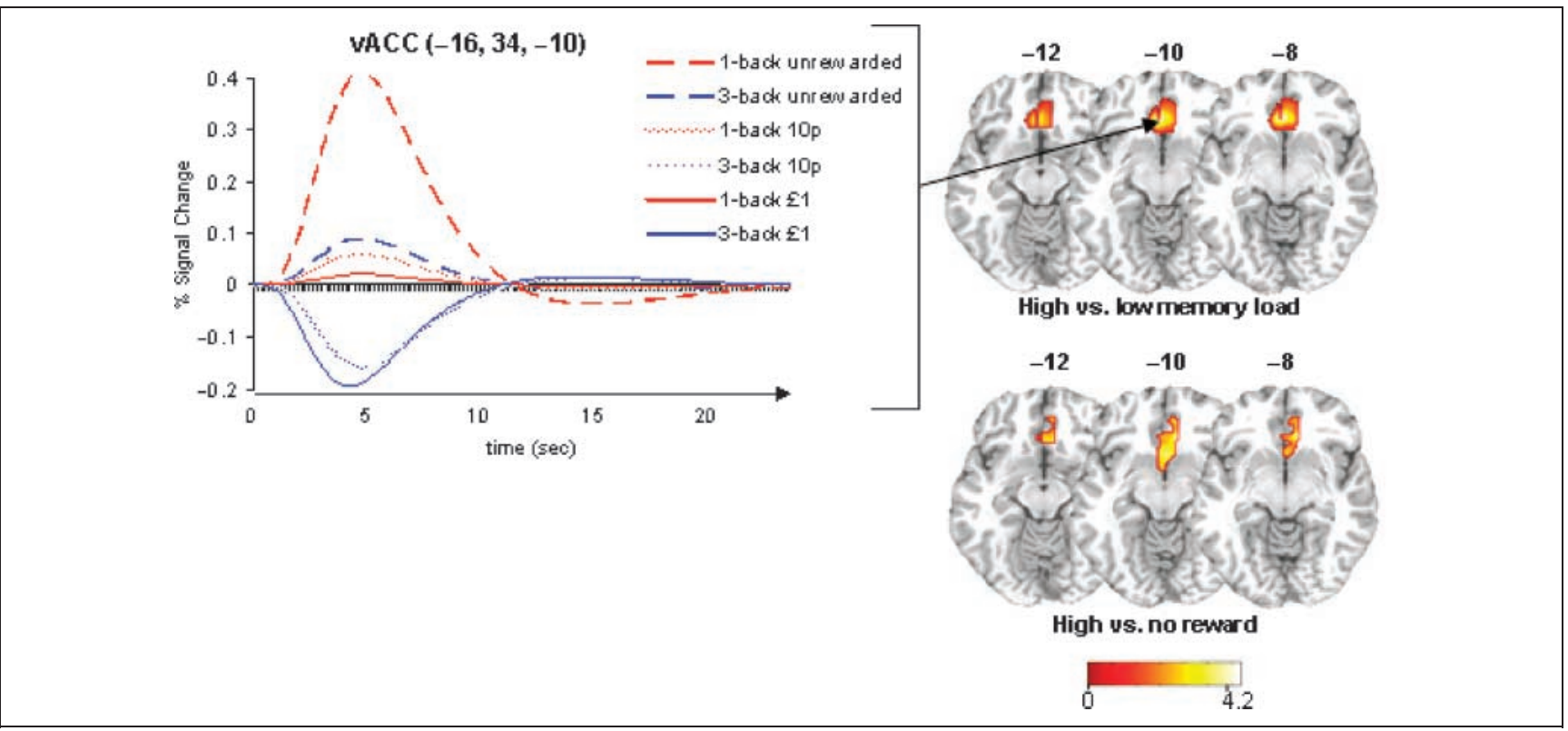

Figure 2. Series of axial slices displaying reduced vACC (BA 32) activity to memory load (high vs. low load) (16, 34, -10) and reward (high vs. no reward) $(0,31,-8)$. Activations were overlaid on a canonical high-resolution structural image in MNI space (MRIcro software; www.psychology.nottingham.ac.uk/staff/cr1/mricro.html). The peristimulus time plot shows response according to stimulus type (task: 3-back and 1-back; reward level: unrewarded, 10p, and £1) at the peak voxel of vACC activation 16, 34, -10 . The color bar shows statistical $T$ variation. 
Table 2. Brain Areas Activated (A) and Deactivated (B) in the Contrast of: (i) High minus Low Rewards; (ii) High minus No Rewards

\begin{tabular}{|c|c|c|c|c|c|}
\hline Region & Cluster Size & p-Corrected & Brodmann's Area & Z Score & $x, y, z$ \\
\hline \multicolumn{6}{|c|}{$i$-(A) High vs. Low Reward Activation } \\
\hline $\mathbf{R} \mathbf{d A C C}$ & 8003 & $<.001$ & BA $32 / 24$ & 4.59 & $24,8,35$ \\
\hline R Lateral PFC & & & BA 44 & 4.56 & $36,13,18$ \\
\hline R dACC & & & BA 24 & 4.48 & $16,10,36$ \\
\hline R LatFPC & 231 & .021 & BA 10 & 3.68 & $24,51,10$ \\
\hline R DLPFC & & & BA 46 & 3.66 & $30,35,6$ \\
\hline L DLPFC & 35 & $.015^{*}$ & BA 46 & 3.35 & $-38,31,6$ \\
\hline \multicolumn{6}{|c|}{ ii-(A) High vs. No Reward Activation } \\
\hline R Caudate & 48 & $.03 *$ & & 4.01 & $20,-11,-23$ \\
\hline \multicolumn{6}{|c|}{ ii-(B) High vs. No Reward Deactivation } \\
\hline R vACC & 71 & $.013^{*}$ & BA 32 & 4.12 & $16,34,-10$ \\
\hline \multirow[t]{2}{*}{ R VMPFC } & & & BA 11 & & $22,38,-16$ \\
\hline & & & BA 11 & & $14,44,-14$ \\
\hline R Subgenual cingulate & 8 & $.033^{*}$ & BA 25 & 3.15 & $4,18,-12$ \\
\hline
\end{tabular}

Clusters significant at $p<.05$ after statistical correction are reported. Multiple peaks within an extent are shown on subsequent lines.

Coordinates are presented in Talairach space. $\mathrm{L}=$ left; $\mathrm{R}=$ right.

*Significant corrected $p$ values shown after SVC.

and high rewarded blocks. Both no-reward compared to high-reward conditions revealed right-sided vACC (BA 32) deactivation spreading to the VMPFC (BA 10), in addition to subgenual cingulate (BA 25) deactivation (Table 2; Figure 2). There were no significant activations detected in the prespecified network of interest for the contrast of both low rewards compared to high rewards.

\section{Interaction Analysis}

One of the key subtractive analysis contrasts is the interaction between memory load (high/low) and reward (high/none). Based on previous studies investigating reward and cognition interactions, we predicted that the high rewarded 3-back task compared to 1-back would more strongly activate LatFPC and DLPFC areas than the unrewarded 3-back compared to 1-back task. This interaction produced significant activation (with SVC) in the right lateral PFC (BA 44), the right DLPFC (BA 9), and the left dACC (BA 24) (Figure 3). The activation in the DLPFC (BA 9) is the only activation not present in the simple main effects and represents a crossover interaction where motivation and cognition interact; a plot of the individual conditions at the peak voxel $[32,41,38]$ supports this claim (Figure 3). Activation in the lateral PFC (BA 44) was differently influenced as the results of the simple main effects suggest that it was driven by an increased

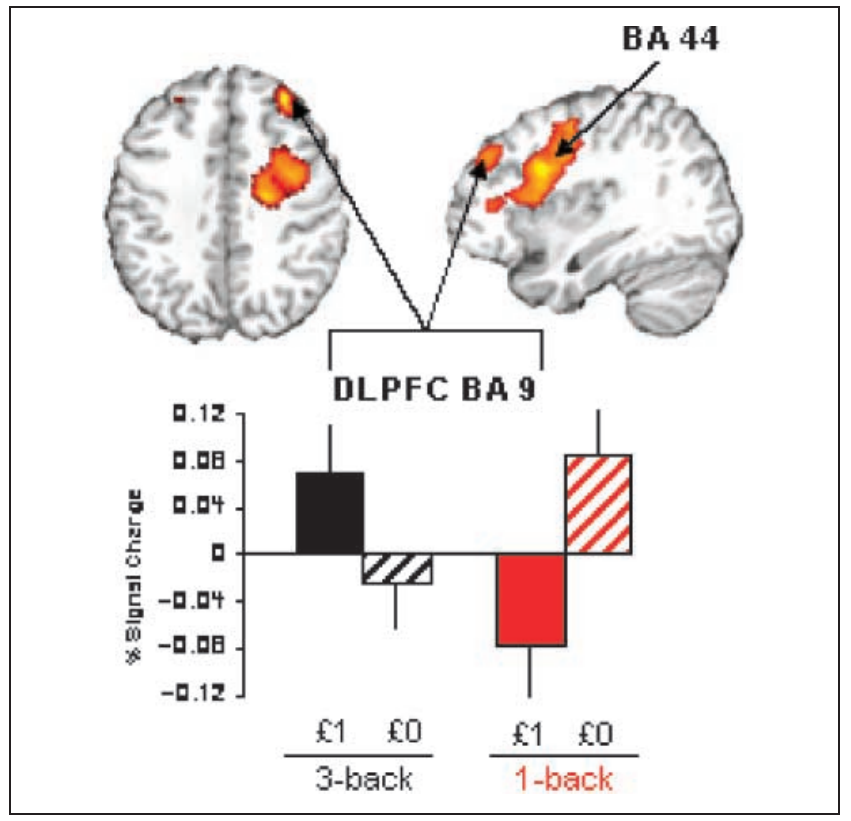

Figure 3. Axial and sagittal slices displaying DLPFC (BA 9) and lateral PFC (BA 44) activation for the interaction of memory load by reward (high reward 3-back vs. 1-back relative to unrewarded 3-back vs. 1-back). Activations were overlaid on a canonical high-resolution structural image in MNI space (MRIcro software; www.psychology. nottingham.ac.uk/staff/cr1/mricro.html). The bar plot shows the strength of activation (beta values) for each individual condition for the crossover interaction at the peak right DLPFC voxel $[32,41,38]$. 
Table 3. Brain Regions Showing Functional Connectivity in the Context of: (i) High vs. Low Memory Load with the LatFPC; (ii) High vs. Low Reward with the Left LatFPC and the Right PFC

\begin{tabular}{|c|c|c|c|c|c|c|}
\hline Region & Cluster Size & Brodmann's Area & Z Score & $x, y, z$ & $p$ & Correlation Direction \\
\hline \multicolumn{7}{|c|}{ (i) R LatFPC (BA 10/46) 3-back vs. 1-back } \\
\hline \multirow[t]{2}{*}{ L DMFC } & \multirow[t]{2}{*}{538} & BA 9 & 4.92 & $-4,60,34$ & .000 & \multirow[t]{2}{*}{ Negative } \\
\hline & & BA 9 & 3.54 & $-18,56,34$ & .000 & \\
\hline R DMFC & & BA 8 & 3.13 & $12,54,40$ & .001 & \\
\hline L Rostral ACC & 95 & BA 32 & 3.46 & $-12,43,9$ & .000 & Negative \\
\hline R IFG & 368 & BA 45 & 3.37 & $40,34,16$ & .000 & Negative \\
\hline L VMPFC & 37 & BA 11 & 2.90 & $-10,48,-11$ & .002 & Negative \\
\hline \multicolumn{7}{|c|}{ (i) L LatFPC (BA 10/46) 3-back vs. 1-back } \\
\hline L VMPFC & 17 & BA 10 & 3.01 & $-18,50,-4$ & .001 & Negative \\
\hline \multicolumn{7}{|c|}{ (ii) $R$ DLPFC (BA 46) High vs. Low Reward } \\
\hline R dACC & 252 & BA 24 & 3.56 & $22,7,29$ & .000 & Negative \\
\hline L Medial PFC & 214 & BA 10 & 3.05 & $-10,61,21$ & .001 & Negative \\
\hline R Medial PFC & 18 & BA 10 & 2.84 & $12,59,12$ & .002 & Negative \\
\hline
\end{tabular}

Analyses were conducted with the threshold of $p<.005$ (with SVC).

Coordinates are presented in Talairach space. $\mathrm{L}=$ left; $\mathrm{R}=$ right.

response to 3-back high rewards relative to 3-back norewards. Thus, this interaction may indicate that, in the lateral PFC, the effect of memory load was greater in the high reward, compared to the no-reward, condition. No significant foci were noted in the opposite interaction contrast.

\section{fMRI Connectivity Analysis}

Using PPI analysis, we examined whether both the right and left LatFPC (BA 10/46) activation negatively predicts activity in the VMPFC (including the vACC and the subgenual cingulate) in the context of high memory load. We observed this to be the case for both hemispheres, which demonstrated negative functional connectivity with the left VMPFC at BA 11 and BA 10 (Table 3; Figure 4). This was present at a threshold of $p<.005$, uncorrected in both instances (significant with SVC). The right LatFPC also showed a negative functional relationship with the bilateral dorsomedial frontal cortex (DMFC; BA 9, 8), the right inferior frontal gyrus (BA 45), and the left rostral cortex (BA 32), in the context of high memory load.

We also examined whether the context of reward elicited a similar pattern of functional connectivity between lateral PFC regions and the VMPFC (Table 3; Figure 4). In the context of high rewards compared to low rewards, the right DLPFC negatively predicted activity in the dACC and also at the threshold of $p<.005$ in the medial PFC (bilaterally), but not in as ventral a location as we had hypothesized (i.e., VMPFC). We did not observe functional connectivity (either positive or negative) between the left-sided lateral PFC (LatFPC; BA 10/46) and any other brain region.

A final psychophysiological analysis examined the relationship between the lateral PFC and the VMPFC/ subgenual cingulate in the context of both high memory load and high reward (Table 4; Figure 5). We observed that right subgenual cingulate (BA 25) activation positively predicted right DLPFC (BA 9) activation in the context of unrewarded 3-back relative to high reward. The correlation between these regions is a negative correlation, as plotting of the individual parameter estimates reveals that the correlation is less negative than for the reverse contrast (Figure 5) of high-rewarded 3back versus unrewarded 3-back. We also investigated whether a seed voxel based on the right lateral PFC (BA 44) region predicted activity anywhere in the VMPFC/ subgenual cingulate in the context of both high reward and high memory load. We observed negative functional connectivity between the right lateral PFC and the right caudate nucleus, and also with the right VMPFC (BA 11) and subgenual cingulate (BA 25) gyrus.

\section{Physiophysiological Interactions}

To examine dorsal striatal modulation of the LatFPCVMPFC pathway, physiophysiological interaction anal- 


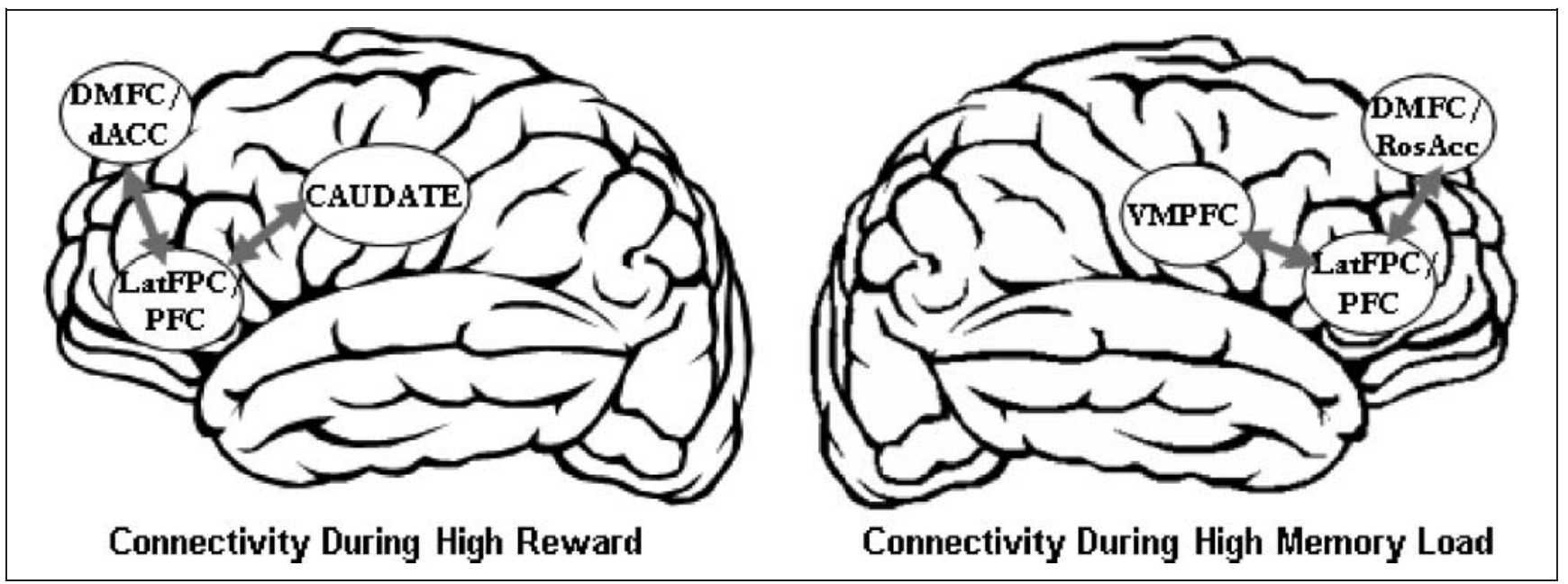

Figure 4. Functional connectivity between the LatFPC/PFC and the VMPFC and other regions of interest (caudate, DMFC, dACC) during high reward relative to low reward and high memory load relative to low. Arrows represent negative functional connectivity. In the context of high memory load versus low, we observed negative connectivity between the bilateral LatFPC and the left VMPFC. A negative relationship was also observed between the right LatFPC and the bilateral dorsomedial PFC and left rostral anterior cingulate. In comparison, the context of high reward did not elicit functional connectivity between the LatFPC/PFC and the VMPFC. Negative connectivity was also detected between the right DLPFC and the bilateral dACC as well as the medial PFC. MFC = medial frontal cortex; RosAcc $=$ rostral anterior cingulate.

yses examined how the interaction between the right LatFPC and the right caudate nucleus covaried with the VMPFC. The LatFPC-caudate interaction showed negative covariation with the right VMPFC (BA 10) and the midline subgenual cingulate (BA 25), significant with SVC.

We also tested whether the dACC modulated the LatFPC-VMPFC pathway using a physiophysiological interaction analyses, which examined how the interaction between the right LatFPC and the dACC covaried with the VMPFC. The LatFPC-dACC interaction showed positive covariation with two medial frontal cortex clusters, one cluster was located in the right medial PFC (BA 10), spreading posteriorly to the midline ACC
(BA 32), and the second in the left VMPFC (BA 10) (Table 5).

\section{DISCUSSION}

The current study examined the relationship between reward processing and working memory load in PFC networks. The hypothesis that inverse neural connections between lateral PFC and VMPFC regions facilitate this relationship was confirmed by PPI analyses, although this relationship was only present in certain experimental contexts. We additionally observed that the relationship between the lateral PFC and VMPFC is influenced

Table 4. Brain Regions Showing Functional Connectivity in the Context of High Memory Load and High Reward, Firstly with the vACC and Secondly with the Right PFC

\begin{tabular}{|c|c|c|c|c|c|c|}
\hline Region & Cluster Size & Brodmann's Area & Z Score & $x, y, z$ & $p$ & Correlation Direction \\
\hline \multicolumn{7}{|c|}{ R Subgenual Cingulate (3-back No Reward vs. High Reward) } \\
\hline R DLPFC & 365 & BA 9 & 3.40 & 38,1929 & .000 & Positive \\
\hline \multicolumn{7}{|c|}{$R$ Lateral PFC (3-back High Reward vs. No Reward) } \\
\hline R Caudate & 7 & & 3.73 & $12,24,2$ & .000 & Negative \\
\hline R VMPFC & 16 & BA 11 & 2.83 & $10,34,-17$ & .002 & Negative \\
\hline R Subgenual cingulate & & BA 25 & 2.60 & $12,28,-16$ & .003 & \\
\hline R VMPFC & 14 & BA 11 & 2.94 & $2,32,-22$ & .002 & Negative \\
\hline
\end{tabular}

Analyses were conducted with the threshold of $p<.005$ (with SVC).

Coordinates are presented in Talairach space. $\mathrm{R}=$ right. 


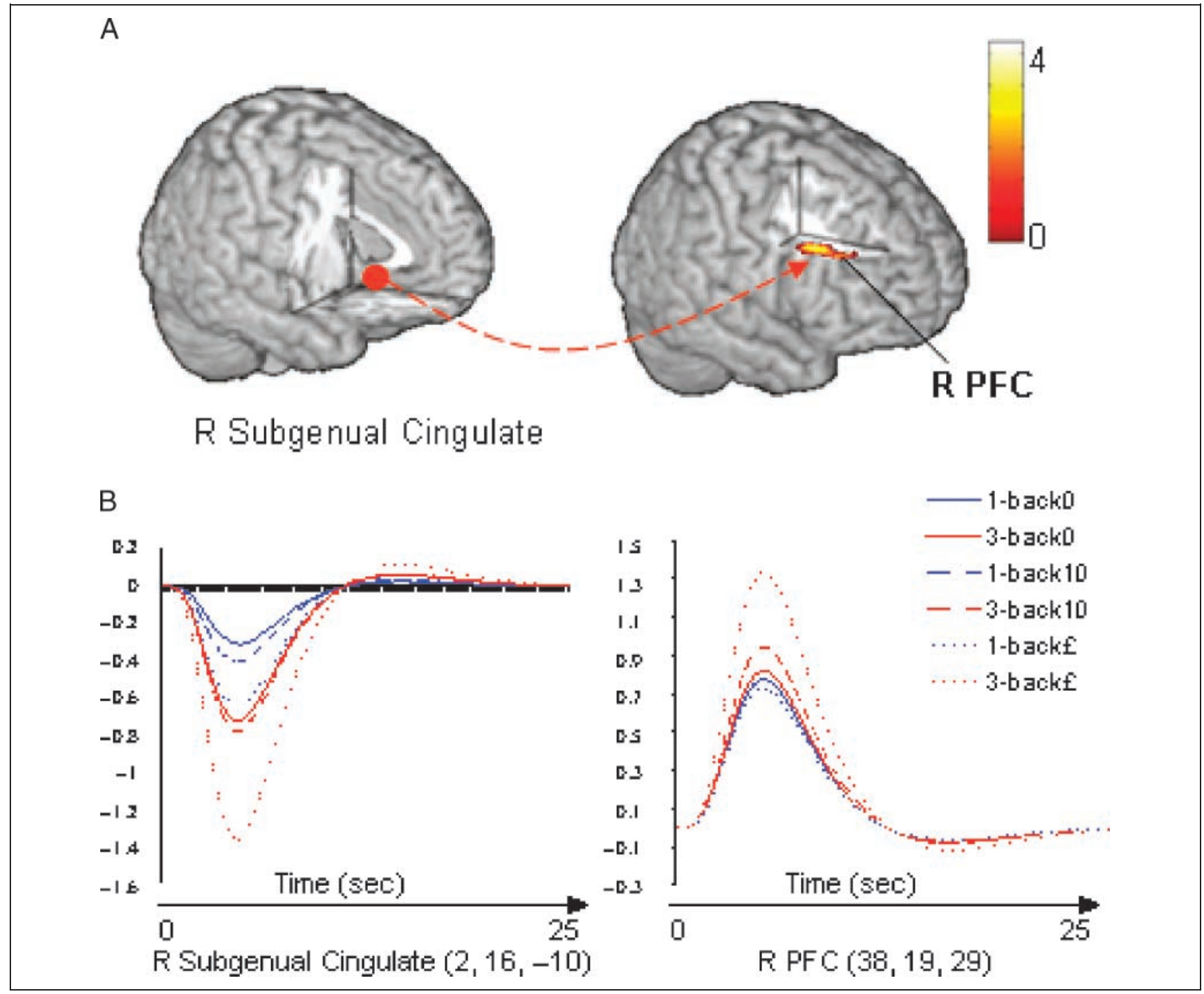

Figure 5. (A) The time series extracted from the subgenual cingulate $[2,16,-10]$ positively predicts right DLPFC (BA 9) activity in the context of 3-back no reward versus 3-back high reward. The color bar shows statistical $T$ variation. (B) The peristimulus time plot shows response according to stimulus type (task: 3-back and 1-back; reward level: unrewarded, 10p, and \&1) at the peak voxel of activation for the subgenual cingulate and the right DLPFC.

Table 5. Physiophysiological Interactions between the Right LatFPC and the Caudate and Also between the Right LatFPC and the Right dACC

\begin{tabular}{|c|c|c|c|c|c|c|}
\hline Region & Cluster Size & Brodmann's Area & Z Score & $x, y, z$ & $p$ & Correlation Direction \\
\hline \multicolumn{7}{|l|}{$R$ LatFPC $\times R$ Caudate } \\
\hline Midline subgenual cingulate & 41 & BA 25 & 3.15 & $0,15,-11$ & .001 & Negative \\
\hline Right VMPFC & 39 & BA 10 & 3.70 & $-24,52,-1$ & .001 & Negative \\
\hline \multicolumn{7}{|l|}{$R$ LatFPC $\times R d A C C$} \\
\hline R VMPFC & 244 & BA 10 & 2.81 & $4,55,5$ & .002 & Positive \\
\hline Midline ACC & & BA 32 & 2.61 & $0,37,4$ & .002 & \\
\hline L VMPFC & 39 & BA 10 & 2.56 & $-2,46,-9$ & .005 & Positive \\
\hline
\end{tabular}

Analyses were conducted with the threshold of $p<.005$ (with SVC).

Coordinates are presented in Talairach space. $\mathrm{L}=$ left; $\mathrm{R}=$ right. 
by connections with the dACC, and through corticalsubcortical connections with the dorsal striatum.

Prior to carrying out the PPI analysis, initial averaged contrasts confirmed that lateral PFC activation was elicited by increasing working memory load, (see also Gilbert \& Fiez, 2004; Pochon et al., 2002). High memory load (3-back task) activated a network of frontal regions including the DLPFC (BA 9/46) and the bilateral frontopolar cortex extending caudally to the lateral PFC (BA 10/ 46). Motivational context also engaged lateral prefrontal areas with high reward activating the right lateral PFC (BA 44), the LatFPC (BA 10/46), and the left DLPFC (BA 46). Additionally, examination of the individual task types showed the DLPFC and the lateral PFC (bilateral BA 44; right BA 46) activation to the high-rewarded 3-back task but not to the high-rewarded 1-back task, showing that lateral PFC areas are engaged most strongly when a task is both highly demanding and strongly motivating, suggesting an integratory role for the region (see also Taylor et al., 2004).

Evidence for an integrative role for the lateral PFC was also provided by the interaction contrast between memory load and reward (high reward 3-back compared to 1-back vs. unrewarded 3-back compared to 1-back). The interaction contrast revealed increased right DLPFC (BA 9) activation, representing a crossover interaction for motivation and cognition, as the plot of the individual parameter estimates revealed. Right lateral PFC (BA 44) activation was also observed, although this was not a true crossover interaction, as activity was observed in the contrast of 3-back high rewards relative to 3-back no-rewards.

Crucially, we observed a consistent pattern of reduced vACC activation to both increases in memory load and reward level. This decrease was more extensive for the main effect of reward (high vs. no-reward) with reduced activation also observed in the VMPFC and the subgenual cingulate gyrus. Reduced VMPFC/vACC activation reflected task performance, which was more accurate to the high-reward conditions and contributes to evidence that increased task demands require the suppression of activity in medial PFC areas in order to minimize competing emotional responses that could interfere with performance (see e.g., Pochon et al., 2002; Raichle et al., 2001).

The critical analyses were the PPI analyses and, in agreement with our hypotheses, we detected negative functional connectivity between both right and left LatFPC regions and the left VMPFC (BA 10,11) to high memory load (high vs. low memory load). The prediction of activity in one brain region by activity in another implies a causal relationship between the two regions (Fletcher et al., 1999; Friston et al., 1997), thus the PPI analyses provide tangible evidence for a dynamic interplay between regions responsible for cognition and motivation. Although the physiological role of this relationship is uncertain, psychologically, it must play a role in the continuous evaluation of an individual's emotional and cognitive needs and alteration of either process in response to current environment. The strongest clues to the function of this relationship come from neuropsychiatry literature, where increased subgenual cingulate activity and decreased DLPFC activity in the resting brain of depressed individuals has been reported (most prominently, Mayberg et al., 1999; see also Drevets, 1999, 2000; Buchsbaum et al., 1997; Soares \& Mann, 1997; Bench, Friston, Brown, Frackowiak, \& Dolan, 1993). Consequently, theories of mood disorders have suggested that cognitive-emotional interactions are mediated via reciprocal PFC and VMPFC connections, and that this relationship is altered in depression (Mayberg et al., 1999). Thus, an impairment in the reciprocal modulation of the lateral PFC on the VMPFC would account for both cognitive deficits (e.g., impairments in memory and attention) and emotional deficits (e.g., negative affect, lack of motivation) commonly observed in major depression (Phillips et al., 2003; Austin et al., 2001).

The averaged contrasts revealed an increase in lateral PFC activity for the main effect of high versus low reward, but a corresponding decrease in VMPFC activity was not evident. Therefore, unsurprisingly, we did not observe functional connectivity between the lateral PFC and VMPFC activation in the context of high rewards (high vs. low reward). However, the combination of both high reward and high memory load elicited the predicted pattern of functional connectivity, with a negative correlation between the VMPFC/subgenual cingulate (BA 11, 25) and the right lateral PFC/DLPFC (BA 44, 9) for high-rewarded 3-back versus unrewarded 3-back task. Consequently, this finding further strengthens the argument that performance in highly demanding cognitive tasks depends on the elimination of concurrent and, possibly interfering, emotional processing. Thus, functional connectivity between the VMPFC and the lateral PFC is context dependent and not a function of increased reward level per se, but is mediated by the allocation of increased attentional/cognitive processes.

The negative functional connectivity between lateral and medial PFC regions extended spatially to include the DMFC (BA 8, 9) during high memory load and also the medial PFC (BA 10) during high reward. These findings suggest that a much broader extent of the medial frontal cortex has an inverse relationship with the lateral PFC than we had predicted, although they fit with previous work showing that the DMFC displays reduced activation during attention-demanding cognitive tasks (Raichle et al., 2001; Shulman et al., 1997). Future studies may wish to consider more dorsal regions of the medial PFC when investigating the interaction between motivation and cognition.

An additional aim of the present study was to assess whether connections with other cortical regions impact on the relationship between lateral and VMPFC regions. This was confirmed with the observation of a negative 
functional relationship between the right LatFPC and the dACC in the context of high reward. As mentioned above, the dACC facilitates appropriate responses and suppresses inappropriate ones (Davidson et al., 2002; Posner \& Raichle, 1994), thus guiding behavior by integrating information on motivation and influencing the allocation of attention and motor responses (Bush et al., 2000). It is likely that the negative connectivity revealed in the PPI analysis is a function of the role the dACC plays in monitoring response conflicts and inhibition (Bush et al., 2000). Negative functional connectivity may also reflect a top-down influence on the LatFPC and could be the crucial signal that suppresses interfering emotional responses in order to perform an executive task, particularly as functional connectivity was only observed with the dACC in the context of high rewards. Such a functional correlation could play a significant role in influencing the relationship between lateral and medial PFC regions. Additionally, in the physiophysiological interaction analyses, the product of LatFPC and dACC activity positively predicted VMPFC (including vACC) activation. Within the framework of our hypothesis, we suggest that this reflects a modulatory effect of the dACC on the lateral PFC-VMPFC network.

Finally, we also found that the right lateral PFC predicted right caudate activation in the context of high reward. Caudate activation has been linked with anticipation of rewards and a negative functional relationship with the lateral PFC most likely indicates a further inhibition of the emotional signal associated with the contextual value of high rewards. The fact that caudate activation was negatively predicted by the lateral PFC in the context of high versus low reward in the 3-back task, when participants required the most concentration, fits this suggestion. A physiophysiological interaction analysis of the product of the LatFPC and the caudate activity positively predicted VMPFC activation, which could reflect a modulatory effect of caudate activity on the lateral PFC-VMPFC pathway. An alternative interpretation of the interaction could be that LatFPC activation modulates a caudate-VMPFC pathway. However, anatomical connectivity studies have only reported that the caudate interacts with anterior cingulate regions (Lawrence, Sahakian, \& Robbins, 1998; Middleton \& Strick, 1996), and not with the VMPFC region observed here, which corroborates our interpretation.

One limitation of the current study is that motivation was not solely reward based, as the potential for financial loss was also present, which may have impacted on both activations and connectivity patterns reported. Although the activations observed are in line with prior studies examining reward processing, future studies should examine the impact of potential monetary loss separately from reward on VMPFC-lateral PFC connectivity, to test whether the cognition-motivational relationship is influenced to a similar level by different motivational states. Furthermore, it would be of interest to test whether other affective states, such as pain, influence the relationship between cognitive and motivational regions, especially as there is evidence of a reduction in regional cerebral blood flow in the VMPFC during the anticipation of pain (Simpson, Drevets, et al., 2001; Hsieh, Stone-Elander, \& Ingvar, 1999). This reduction could reflect coping strategies to reduce anxiety (Simpson, Drevets, et al., 2001), consistent with a role for the vACC in the regulation of emotional responses. We would therefore predict a functional relationship between VMPFC areas and the lateral PFC, where attentional or cognitive processing may influence pain.

To summarize, we have presented a novel approach to the analysis of the interaction between motivational and cognitive processing. This approach involved examining VMPFC and lateral PFC areas as a dynamic integrated system by exploring interregional modulations of these regions. We provide converging evidence that VMPFC and lateral PFC areas interact in an inverse relationship that is context dependent. We found that connectivity between the VMPFC-lateral PFC pathway is modulated as a function of high memory load, or by high memory load in tandem with a highly motivating context, but not by a motivating context per se. Additionally, we observed that the dACC and the caudate nucleus modulate the lateral PFC-VMPFC pathway. The present results provide direct support for theories of mood regulation (Mayberg et al., 1997, 1999), and suggest that the complementary interaction of the medial and lateral PFC observed may be dysfunctional in mood disorders such as major depression.

\section{Acknowledgments}

This research was funded by grants from the EPSRC, the British Academy, and also by the Lord Dowding Fund.

Reprint requests should be sent to Olivia Longe, Neurosciences, School of Life \& Health Sciences, Aston University, Birmingham B4 7ET, UK, or via e-mail: o.a.longe@aston.ac.uk.

\section{REFERENCES}

Alexander, G. E., Crutcher, M. D., \& DeLong, M. R. (1990). Basal ganglia-thalamocortical circuits: Parallel substrates for motor, oculomotor, "prefrontal" and "limbic" functions. Progress in Brain Research, 85, 119-146.

Austin, M. P., Mitchell, P., \& Goodwin, G. M. (2001). Cognitive deficits in depression: Possible implications for functional neuropathology. British Journal of Psychiatry, 178, 200-206.

Beck, A. T., Ward, C. H., Mendelson, M., Mock, J., \& Erbaugh, J. (1961). An inventory for measuring depression. Archives of General Psychiatry, 4, 561-571.

Bench, C. J., Friston, K. J., Brown, R. G., Frackowiak, R. S., \& Dolan, R. J. (1993). Regional cerebral blood flow in depression measured by positron emission tomography: 
The relationship with clinical dimensions. Psychological Medicine, 23, 579-590.

Berns, G. S., McClure, S. M., Pagnoni, G., \& Montague, P. R. (2001). Predictability modulates human brain response to reward. Journal of Neuroscience, 21, 2793-2798.

Brett, M., Christoff, K., Cusack, R., \& Lancaster, J. (2001). Using the Talairach atlas with the MNI template. Neuroimage, 13, S85.

Buchsbaum, M. S., Wu, J., Siegel, B. V., Hackett, E., Trenary, M., Abel, L., et al. (1997). Effect of sertraline on regional metabolic rate in patients with affective disorder. Biological Psychiatry, 41, 15-22.

Bush, G., Luu, P., \& Posner, M. I. (2000). Cognitive and emotional influences in anterior cingulate cortex. Trends in Cognitive Sciences, 4, 215-222.

Das, P., Kemp, A. H., Liddell, B. J., Brown, K. J., Olivieri, G., Peduto, A., et al. (2005). Pathways for fear perception: Modulation of amygdala activity by thalamo-cortical systems. Neuroimage, 26, 141-148.

Davidson, R. J., Pizzagalli, D., Nitschke, J. B., \& Putnam, K. (2002). Depression: Perspective from affective neuroscience. Annual Review of Psychology, 53, 545-574.

Delgado, M. R., Locke, H. M., Stenger, V. A., \& Fiez, J. A. (2003). Dorsal striatum responses to reward and punishment: Effects of valence and magnitude manipulations. Cognitive, Affective \& Behavioral Neuroscience, 3, 27-38.

Delgado, M. R., Nystrom, L. E., Fissell, C., Noll, D. C., \& Fiez, J. A. (2000). Tracking the hemodynamic responses to reward and punishment in the striatum. Journal of Neurophysiology, 84, 3072-3077.

Drevets, W. C. (1999). Prefrontal cortical-amygdalar metabolism in major depression. Annals of the New York Academy of Sciences, 877, 614-637.

Drevets, W. C. (2000). Functional anatomical abnormalities in limbic and prefrontal cortical structures in major depression. Progress in Brain Research, 126, 413-431.

Egner, T., \& Hirsch, J. (2005). The neural correlates and functional integration of cognitive control in a Stroop task. Neuroimage, 24, 539-547.

Elliott, R., Friston, K. J., \& Dolan, R. J. (2000). Dissociable neural responses in human reward systems. Journal of Neuroscience, 20, 6159-6165.

Elliott, R., Newman, J. L., Longe, O. A., \& Deakin, J. F. (2003). Differential response patterns in the striatum and orbitofrontal cortex to financial reward in humans: A parametric functional magnetic resonance imaging study. Journal of Neuroscience, 23, 303-307.

Fletcher, P. J., McKenna, K. J., Friston, C. D., \& Dolan, R. J. (1999). Abnormal cingulate modulation of fronto-temporal connectivity in schizophrenia. Neuroimage, 9, 337-342.

Friston, K. J., Büchel, C., Fink, G. R., Morris, J., Rolls, E., \& Dolan, R. J. (1997). Psychophysiological and modulatory interactions in neuroimaging. Neuroimage, 6, 218-229.

Gilbert, A. M., \& Fiez, J. A. (2004). Integrating rewards and cognition in the frontal cortex. Cognitive, Affective $\&$ Behavioral Neuroscience, 4, 540-552.

Gray, J. R., Braver, T. S., \& Raichle, M. E. (2002). Integration of emotion and cognition in the lateral prefrontal cortex. Proceedings of the National Academy of Sciences, U.S.A., 99, 4115-4120.

Groenewegen, H. J., \& Uylings, H. B. (2000). The prefrontal cortex and the integration of sensory, limbic and autonomic information. Progress in Brain Research, 126, 3-28.

Hsieh, J. C., Stone-Elander, S., \& Ingvar, M. (1999). Anticipatory coping of pain expressed in the human anterior cingulate cortex: A positron emission tomography study.

Neuroscience Letters, 262, 61-64.
Knutson, B., Adams, C. M., Fong, G. W., \& Hommer, D. (2001). Anticipation of increasing monetary reward selectively recruits nucleus accumbens. Journal of Neuroscience, 21, R159.

Lauwereyns, J., Takikawa, Y., Kawagoe, R., Kobayashi, S., Koizumi, M., Coe, B., et al. (2002). Feature-based anticipation of cues that predict reward in monkey caudate nucleus. Neuron, 33, 463-473.

Lawrence, A. D., Sahakian, B. J., \& Robbins, T. W. (1998). Cognitive functions and corticostriatal circuits: Insights from Huntington's disease. Trends in Cognitive Sciences, 2, 379-388.

Leh, S. E., Ptito, A., Chakravarty, M. M., \& Strafella, A. P. (2007). Fronto-striatal connections in the human brain: A probabilistic diffusion tractography study. Neuroscience Letters, 419, 113-118.

Mayberg, H. S. (1997). Limbic-cortical dysregulation: A proposed model of depression. Journal of Neuropsychiatry and Clinical Neurosciences, 9, 471-481.

Mayberg, H. S., Liotti, M., Brannan, S. K., McGinnis, S., Mahurin, R. K., Jerabek, P. A., et al. (1999). Reciprocal limbic-cortical function and negative mood: Converging PET findings in depression and normal sadness. American Journal of Psychiatry, 156, 675-682.

McIntosh, A. R., Rajah, M. N., \& Lobaugh, N. J. (1999). Interactions of prefrontal cortex in relation to awareness in sensory learning. Science, 284, 1531-1533.

Middleton, F. A., \& Strick, P. L. (1996). The temporal lobe is a target of output from the basal ganglia. Proceedings of the National Academy of Sciences, U.S.A., 93, 8683-8687.

Phillips, M. L., Drevets, W. C., Rauch, S. L., \& Lane, R. (2003). Neurobiology of emotion perception: II. Implications for major psychiatric disorders. Biological Psychiatry, 54, 515-528.

Pochon, J. B., Levy, R., Fossati, P., Lehericy, S., Poline, J. B., Pillon, B., et al. (2002). The neural system that bridges reward and cognition in humans: An fMRI study. Proceedings of the National Academy of Sciences, U.S.A., 99, 5669-5674.

Posner, M. I., \& Raichle, M. E. (1994). Images of mind. New York: Scientific American Books.

Raichle, M. E., MacLeod, A. M., Snyder, A. Z., Powers, W. J., Gusnard, D. A., \& Shulman, G. L. (2001). A default mode of brain function. Proceedings of the National Academy of Sciences, U.S.A., 98, 676-682.

Schultz, W., Tremblay, L., \& Hollerman, J. R. (2000). Reward processing in primate orbitofrontal cortex and basal ganglia. Cerebral Cortex, 10, 272-283.

Shulman, G. L., Corbetta, M., Buckner, R. L., Raichle, M. E., Fiez, J. A., Miezin, F. M., et al. (1997). Top-down modulation of early sensory cortex. Cerebral Cortex, 3, 193-206.

Simpson, J. R., Jr., Drevets, W. C., Snyder, A. Z., Gusnard, D. A., \& Raichle, M. E. (2001). Emotion-induced changes in human medial prefrontal cortex: II. During anticipatory anxiety. Proceedings of the National Academy of Sciences, U.S.A., 98, 688-693.

Simpson, J. R., Jr., Snyder, A. Z., Gusnard, D. A., \& Raichle, M. E. (2001). Emotion-induced changes in human medial prefrontal cortex: I. During cognitive task performance. Proceedings of the National Academy of Sciences, U.S.A., 98, 683-687.

Soares, J. C., \& Mann, J. J. (1997). The functional neuroanatomy of mood disorders. Journal of Psychiatric Research, 31, 393-432.

Taylor, S. F., Welsh, R. C., Wager, T. D., Phan, K. L., Fitzgerald, K. D., \& Gehring, W. J. (2004). A functional neuroimaging 
study of motivation and executive function. Neuroimage, 21, 1045-1054.

Watanabe, M., Hikosaka, K., Sakagami, M., \& Shirakawa, S. (2002). Coding and monitoring of motivational context in the primate prefrontal cortex. Journal of Neuroscience, 22, 2391-2400.

White, N. S., \& Alkire, M. T. (2003). Impaired thalamocortical connectivity in humans during general-anesthetic-induced unconsciousness. Neuroimage, 2, 402-411.

Williams, L. M., Das, P., Liddell, B. J., Kemp, A. H., Rennie, C. J., \& Gordon, E. (2006). Mode of functional connectivity in amygdala pathways dissociates level of awareness for signals of fear. Journal of Neuroscience, 26, 9264-9271.

Worsley, K. J., Marrett, S., Neelin, P., Vandal, A. C., Friston, K. J., \& Evans, A. C. (1996). A unified statistical approach for determining significant signals in images of cerebral activation. Human Brain Mapping, 4, 58-73.

Yeterian, E. H., \& Pandya, D. N. (1991). Prefrontostriatal connections in relation to cortical architectonic organization in rhesus monkeys. Journal of Comparative Neurology, 312, 43-67. 